\title{
Access Control in Shared Access Networks Supporting Internet DiffServ
}

\author{
J. D. Angelopoulos, N. Leligou, Th. Orphanoudakis, G. Pikrammenos, \\ J. Sifnaios and I. S. Venieris
}

National Technical University of Athens, Electrical and Computer Engineering Department, GR15753, Athens, Greece, e-mail: jangel@cs.ntua.gr

Key words: $\quad$ MAC, HFC, PON, DiffServ, QoS.

\begin{abstract}
Shared access networks such as hybrid fiber/coaxial and passive optical networks have emerged as promising ways to reduce the cost of the transition to a broadband access infrastructure and provide a graceful upgrade path towards the photonization of the local loop. The TDMA multiplexing of traffic entering such a system is governed by the MAC (Medium Access Control) protocol, which arbitrates the allocation of bandwidth to the shared feeder. At the same time the need to integrate telecom services presenting demanding quality requirements with plain Internet best-effort services over the same infrastructure, brings new issues to the design of such an access mechanism. The MAC protocol as the only arbiter of the upstream bandwidth directly affects the Quality of Service (QoS) provided to each upstream traffic flow and must meet several constraints. Such constraints include the adequate speed of operation exploiting in the highest degree the speed of $\mathrm{H} / \mathrm{W}$ implementation, flexibility to support efficiently the largest number of services and applications offering an adequate number of QoS classes like the differentiated services (DiffServ)and independence of higher layers, protocols and future extensions to traffic management specifications. The implementation of a mechanism targeting these goals and its evaluation using computer simulation are presented in this paper.
\end{abstract}

The original version of this chapter was revised: The copyright line was incorrect. This has been corrected. The Erratum to this chapter is available at DOI: 10.1007/978-0-387-35410-1_19 


\section{INTRODUCTION}

The access part of telecommunication networks also known as the "local loop" is characterised by low traffic intensities and high cost per traffic volume. It is typically amortized by very few customers and the last drop by just one. Thus it is very sensitive to cost and experiences much longer investment life-cycles. To reduce the cost while retaining the evolutionary prospects, tree-shaped topologies exploiting a shared feeder fiber are a promising solution. Typical examples are bi-directional Hybrid Fiber/Coaxial (HFC) networks (an evolution of CATV systems) as well as passive optical networks (PONs). Both architectures rely on shared optical fibers in the feeder section while the existing copper infrastructure remains on the home side at least for the introductory phase when fiber will not go into the last drops.

In both systems, sharing of the upstream channel (from customer to network) is usually effected through TDMA multiplexing. A Medium Access Control (MAC) protocol is employed to arbitrate the access to the slotted common medium by allocating slots to customer terminations according to demand [5], [6].

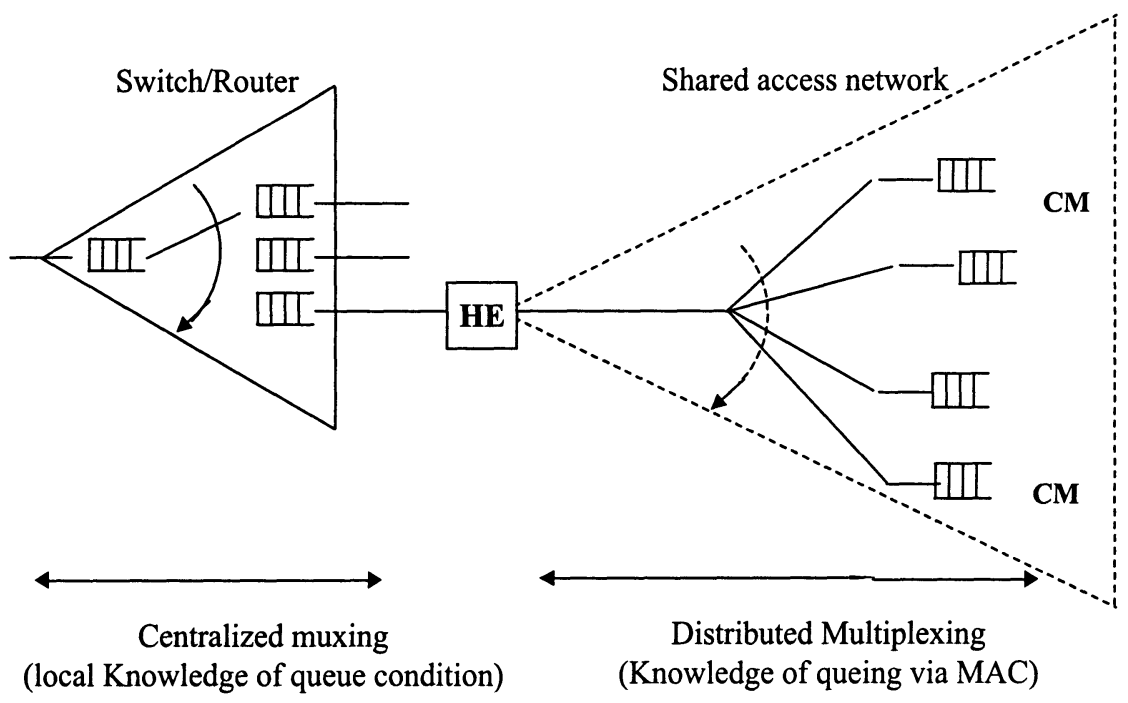

Figure 1. Conceptual view of distributed access multiplexing

This results in a distributed queuing system characterised by the long time required to pass control information from the queuing points to the service controller residing at the head-end. The allocation of upstream slots in a tree-topology access system is based on a reservation method which allows to dynamically adapt the bandwidth distribution to traffic 
fluctuations. The MAC controller collects access requests and then allocates the upstream slots by sending access permits. It is important to note that the service policy of the MAC governs the distributed multiplexing from a central point situated miles away at the head-end. This has the important implication that regarding the delay control, the acquisition of arrival information and buffer fill levels includes a considerable delay element. This delay element does not exist in centralized multiplexing schemes, operating typically at a switch queuing point. In contrast, drop policies must be distributed over all network terminations (cable modems) where the flow identity and fill levels are known. Because of the much larger reservation delay and statistical behavior of the aggregations from many customers, special care must be taken to safeguard QoS to sensitive traffic. This requires a prioritization scheme integrated in the MAC function of PONs and HFC systems as was already identified in [5]. This must be in line with the recent advances in networking that include the two major approaches in offering multi-service broadband access enriched with differentiated QoS support namely the categorization of traffic classes by the ATM Forum [8] and the differentiated services (DiffServ) strategy of IETF [1]. In the following sections the implementation and performance of an HFC MAC aligned to the DiffServ architecture will be presented. Similar design considerations apply to PON MAC protocols with some adaptation to the higher line rates of a PON.

\section{DESIGN OF A MAC FUNCTION FOR A SERVICE INTEGRATED MULTILAYER HFC ACCESS NETWORK}

From a traffic engineering point of view, the tree-shaped access system exhibits a very different behaviour between the upstream (from customers to core) and the downstream (from core to the customers) direction. In the downstream, the broadcast nature of the medium creates replicas of the signal (in the passive splitters of PONs or the taps of HFC systems) giving rise to privacy and security issues. These are typically dealt with by means of encryption, which is out of the scope of this work. In the upstream on the other hand, the MAC control function effects multiplexing / concentration of the traffic from all active modems as typically occurs in the output ports of any switching node (Figure 1). However in this case it is characterized by the distributed nature of the queuing points and the additional difficulties in the exchange of control information. Failure to recognize the packet precedence and behave accordingly would result in loss of QoS for any sensitive flow under high load. To apply any scheduling or priority 
discipline requires the correlation of the traffic from all multiplexed sources going to the one common egress point of the system.

Traffic categorization may be connection based following the ATM approach or flow based following the DiffServ approach of the IETF. The system under consideration employs a TDMA slotting designed for ATM cells. Such an ATM slotting complies with the approach followed by the main standards bodies, like DAVIC 1.3 and 1.4, DVB/ETSI ETS 300 800, (now incorporated in DAVIC), and IEEE 802.14 [4]. Regarding PONs the ATM slot size is used in G.983 [9]. So we will consider the ATM size slots as a quantum of the MAC assigned bandwidth allocation. To accommodate IP frames, several slots are successively assigned by the MAC. However, even if IP is not offered over ATM, the legacy of short slots can be used to advantage in the context of the DiffServ architecture over a slow shared link such as HFC. Namely, it allows to suspend the transmission of a lower priority packet, on the boundary of a slot (cell), and transmit delay-sensitive packets before resuming the transmission of the low priority one. In addition, fixed slots are easier to handle in a H/W based MAC.

Logically the MAC protocol functions similarly to a centralized multiplexer i.e. connections or IP flows with demanding QoS must be identified and receive properly differentiated treatment from the plain best effort traffic. This is accomplished in the cable modem or the ONU during packet classification by placing the corresponding cells in the high priority queue which will subsequently place higher priority requests and activate the higher priority permit allocation MAC algorithms.

To reduce complexity in this cost-sensitive residential access system, services are grouped into behavior aggregates (classes) with a similar set of requirements providing scalability and flexibility. Given the fluidity of service class definition and the fact that the MAC is implemented mainly in $\mathrm{H} / \mathrm{W}$, it was deemed adequate to implement just 4 priority classes in the MAC, leaving finer aggregations and more elaborate forwarding policies, to be implemented at the egress of the HFC system. All that is required from the MAC is not to deny quality to any groups of flows. The basis of this approach is the use of access priorities in the reservation system which can be programmed to fit with required traffic descriptors by means of S/W programming of the mapping of flows to priorities.

The characteristics of the four aggregation levels/priorities are the following:

- The high priority is devoted to delay-sensitive periodic CBR traffic.This class targets services with very strict delay requirements, which undergo strict traffic profile control (traffic conditioning) such as CBR ATM connections and the EF (Expedited Forwarding) service [2]. 
- The second priority level is devoted to real-time variable rate flows, such as video services or VoIP and it is provided with a minimum guarantied rate by means of pre-allocated permits and peak rate policing for guaranteed QoS. MAC exercises a policing function by rate checking before issuing the permits. This is based on credit allocation at the time of subscription or connection set-up. In the DiffServ context it could be used for the top AF (Assured Forwarding) class.

- The third priority is devoted to data services with higher requirements than best-effort. The traffic profile control assumed for this class aims at minimizing the loss of packets and the disturbance to other traffic. The credit scheme is used to guarantee a minimum rate (while credits last) while traffic exceeding this limit is relegated to the $4^{\text {th }}$ priority permit generation. The $3^{\text {rd }}$ priority mechanism is suited to the support of all four or the lower three AF classes [3] as well as ABR connections in the ATM context that also need separate queuing and information about buffer levels in order to possibly integrate the closed loop control into the MAC function as described in. (Drop policies can be independently applied at the modem queuing points).

- The fourth priority is reserved for plain best-effort services such the UBR as well as traffic which employ loss based flow control at the TCP level and can be very disruptive to the other classes when sharing the same queue.

The last three priorities employ reservation while the first unsolicited permits. The reservations operate by means of three request fields totaling 8 bits. Two bits are used for the 2nd class and three bits for the other two creating a byte which is piggy-backed in every upstream slot. This byte uses the place of the HEC field of the ATM cell which is not needed for cell delineation since an additional synchronization preamble is employed because of the burst mode operation. The total length of the upstream slot has a size of 64 bytes accommodating except of the cell payload a synchronization preamble. The frame structure in the downstream direction is dictated by several mandates of the physical layer (synchronization, flexibility over many modulation constellations and rates adapting to plant conditions, interleaving, Reed-Solomon FEC etc). These issues are covered in the relevant standards, e.g. [4], and will not be discussed here since we focus on the traffic multiplexing issues. For the operation of the MAC all that is important is that a number of permits is provided in a periodic basis corresponding to the number of upstream slots over the same period.

The reservations in the HFC MAC are usually assisted by contention on special reservation mini-slots [4] in addition to the piggy-backed requests. This is needed for the announcement of the first arrival of a burst since the piggy-back mechanism is not self-starting but relies on the existence of 
previous traffic for the announcement of new arrivals [6]. However the MAC departs from this approach in two points: first it provides for all three simultaneous requests (one from each queue) as explained above, irrespective of which queue provided the cell, and second it employs round robin polling instead of contention. Simple polling is considered adequate since the system targets an advanced stage of deployment when the HFC take-up will be significant and the customer clusters will be smaller with much higher bandwidth needs. In PONs, polling is preferred because there is a high number of slots contained in the round trip making collision resolution impractical.

The multiple requests are the tool for the higher QoS capabilities of the system. They are necessary if higher priority traffic is to be quickly made known to the head-end. This feature enables the algorithm at the head-end to offer precedence in the permit allocation to the high priority on a global basis and not just among the cells of the same termination, which is of a very limited value.

\section{IMPLEMENTATION OF THE MAC CONTROLLER}

The MAC controller was implemented on the Access Network Adaptation board. Since the MAC considered a sub-layer of the physical layer and is closely related to the physical layer functions (TDMA, framing, etc.) the permit generation function was selected to be integrated with the downstream framer component, which was responsible for the construction of the downstream frame including the transmission permits. The whole design was programmed and placed on a Field Programmable Gate Array (FPGA) chip. This decision made possible the re-design of the implementation to adjust to modified frame structures, rates etc. An embedded processor (On Board Controller) was used to calculate and modify all non-real time parameters such as the programmed (pre-allocated or provisioned) bandwidth distribution, which may vary with time due to the switch on or off of the modems. An external static RAM chip was used for buffering necessary protocol information in addition to the available on-chip memory provided by the FPGA, which was exploited to keep state information and speed-up the permit generation process. The MAC algorithm works as follows:

A list of 512 permits is employed, which has been prepared by the OBC on the basis of subscription data for the scheduling of all pre-arbitrated permits. The OBC executes the Call Admission Control (CAC) scheme and writes periodically permits for the $1^{\text {st }}$ and $2^{\text {nd }}$ priority connections at the peak 
and the minimum guarantied rate accordingly. Permits are also inserted for polling requests by modems that have not established a $1^{\text {st }}$ or $2^{\text {nd }}$ priority connection. A 3-bit field is foreseen in the permit format in order to indicate the queue (and the corresponding QoS class) targeted, facilitating internal calculations. This list is cyclically read out by the MAC controller $\mathrm{H} / \mathrm{W}$, and the permits sent downstream. It worths noting that the format of the permits sent downstream differs from that written in the list in the sense that they include no coloring but only the modem ID and the modem is responsible for selecting the appropriate queue for transmission in an increasing order of priority. Techniques to space the permits in the list are given in [7]. This list is stored on the external SRAM chip. At the end of each cycle, the embedded controller updates also a list of credits for the $2^{\text {nd }}$ and $3^{\text {rd }}$ priority connections. These credits are used for policing $2^{\text {nd }}$ priority connections at the contracted peak rate and guaranteeing the minimum calculated bandwidth of $3^{\text {rd }}$ priority connections as described above. Since ATM signaling is also supported, the permit and credit lists can be updated dynamically to add new connections using a second copy.

The three lower priorities are serviced dynamically on the basis of requests by filling in locations whenever they are left empty in the permit list (representing unallocated bandwidth). The requests per queue/priority arriving at the MAC controller in the head-end, are used to update the 2 outstanding request totals for each modem and queue, by incrementing the relevant counters by the amount of new cell arrivals. At the same time the downstream permit positions in the frame are filled with permits produced by an engine scanning in a round robin fashion the outstanding request counters and reducing them by one for each permit scheduled. The higher priority counters are inspected first and only if all are empty the same process is repeated for the immediately lower priority. To expedite the process, flags are used to quickly detect and skip all empty locations. The MAC H/W subtracts the credits as it issues permits and stops serving any modem queue that exceeded its allocated apportionment. This policing action guarantees that malicious users can not disturb complying traffic. The credit check is used differently in the 3rd priority. When credits are exhausted, left-over requests are added to the $4^{\text {th }}$ priority ones thus a minimum rate is guaranteed but any excess is considered plain best-effort, in accordance with $\mathrm{AF}$ rules. The operation of the permit scheduler and the execution of the MAC algorithm is depicted in Figure 2. 


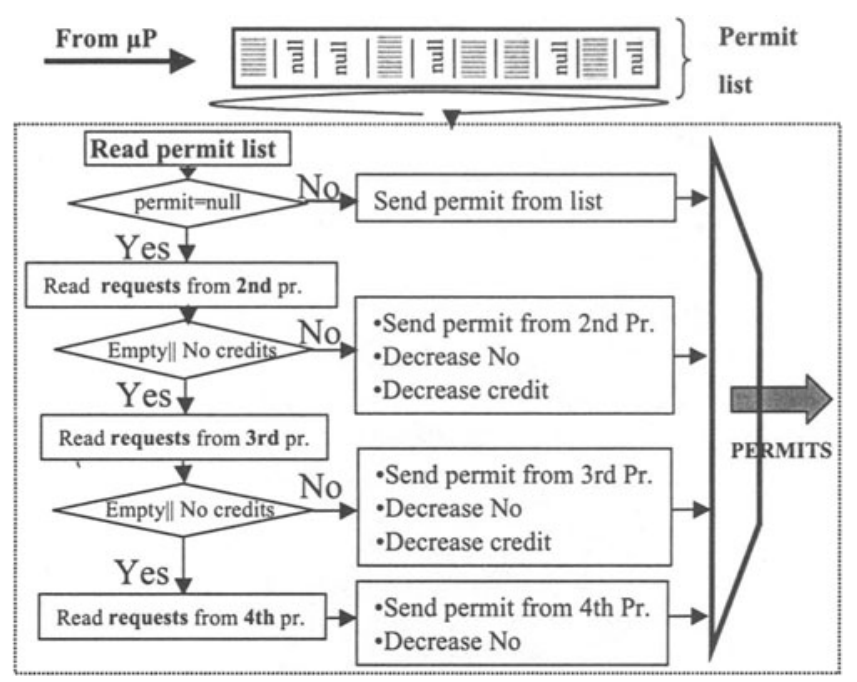

Figure 2. Operation of the permit scheduler.

\section{PERFORMANCE ASSESSMENT}

For the evaluation of the above MAC mechanisms a computer simulation model was created using the PTOLEMY platform for modeling the MAC function under certain loading conditions. The scenarios used 10 cable modems loaded with uniform traffic per priority for each modem. Each source used a common ON-OFF model, generating traffic at the slot/cell level, with on and off periods exponentially distributed. The upstream channel rate was set as peak rate. There was no limit set to credits, since the policing function would only be meaningful to demonstrate with malicious sources that was out of the scope of these tests, since the results are determinist. The duration of the runs was 1.5 million slots and the upstream slot duration was used as a time unit for the simulations (170.6 $\mu \mathrm{s})$.

Figure 3 depicts the probability distribution function (pdf) of the access delay for the three lower priorities $\left(2^{\text {nd }}, 3^{\text {rd }}\right.$, and $\left.4^{\text {th }}\right)$ under a total load of $85 \%$. The $1^{\text {st }}$ priority list was using $10 \%$ of the load for CBR traffic (or virtual leased lines by EF in the DiffServ model of usage), with the other $75 \%$ equally distributed among the other 3 priorities. Only the variable part of the delay is shown, as the fixed round trip time (about 4 slots) required for the modem request (upstream) and the permit response (downstream) is not counted. 


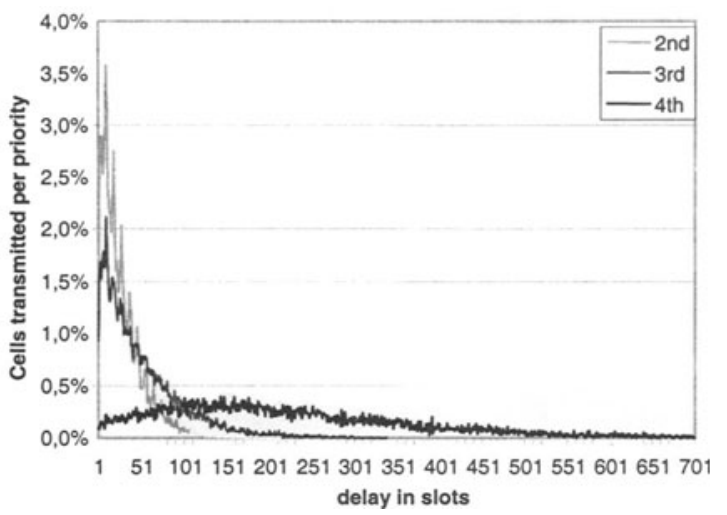

Figure 3. Pdf of access delay (total load 110\%).

The $1^{\text {st }}$ priority is not presented since it exhibits deterministic behaviour, as the transmission permits are periodically pre-programmed in the list [7], and thus, the delay never exceeds the fixed permit distance. The delay advantage provided by the prioritization leads to almost all the slots of the $2^{\text {nd }}$ priority accessing in less than 250 slots (i.e. $43 \mathrm{~ms}$ ) while those of the $3^{\text {rd }}$ in less than 350 slots $(60 \mathrm{~ms})$. Of course there is no bound for the $4^{\text {th }}$ priority which can exceed any limit depending on the total loading.

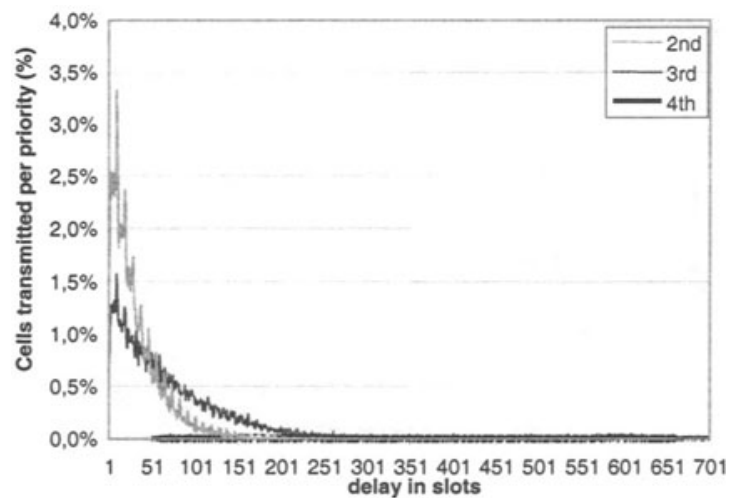

Figure 4. Pdf of access delay (total load $85 \%$ ).

The pdf of delay under a higher offered load of $110 \%$ is shown in Figure 4. The list contained $10 \%$ unsolicited CBR traffic ( $1^{\text {st }}$ priority), as the $2^{\text {nd }}$ and $3^{\text {rd }}$ priority sources offered a load equal to $30 \%$ of capacity, and $40 \%$ the $4^{\text {th }}$. The first two priorities do not exhibit any significant behaviour difference with the previous scenario, since they encounter a lightly loaded medium as before. The effect of prioritization is exactly to hide the presence of any 
lower priority traffic, which is prevented from competing against the protected sensitive traffic. The effect on the $4^{\text {th }}$ priority is very strong since it is throttled down to the $30 \%$ of capacity left over from the other three. The occurred delay distribution is thinly spread till theoretically infinite delay. This can not be shown in the simulation results since buffers overflow no matter how much memory is allocated. The average delay is not bounded for the $4^{\text {th }}$ priority, as shown in Figure 5.

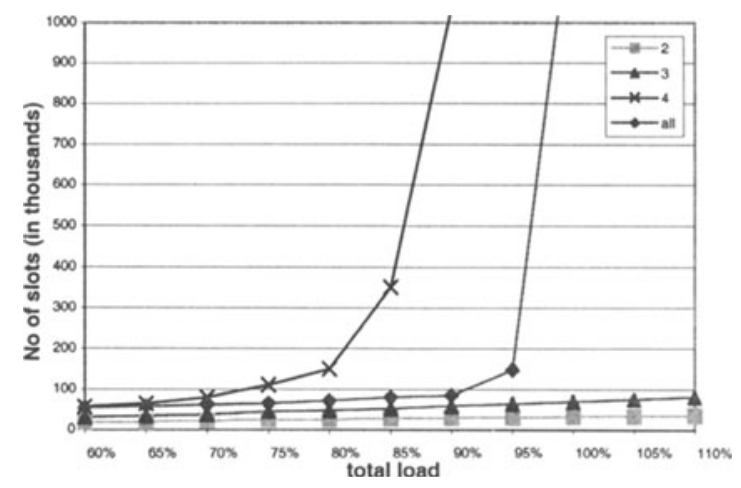

Figure 5. Mean delay vs. total load.

Again $10 \%$ of the upstream bandwidth was pre-allocated by means of programmed permits in the permit list as necessary for the polling of new bursts and the rest equally distributed among the priorities and modems for several values of the total offered load as depicted in Figure 5. The delay for the $2^{\text {nd }}$ and $3^{\text {rd }}$ priorities increases very smoothly with the load leaving the $4^{\text {th }}$ priority to suffer the congestion. The $4^{\text {th }}$ priority delay increases asymptotically towards the $100 \%$ line, as is typical of any queuing system.

At the same figure we can observe the behavior of the system when only two priorities are valid: $1^{\text {st }}$ and "all". The first priority ramains as it was, while the three others are merged to one, the "all". The load balance remains $10 \%$ for the $1^{\text {st }}$ while the "all" priority occupies the missing part of the total load represented at the horizontal axis. It is obvious that the "all" priority behaves better than the $4^{\text {th }}$ priority and worst than the $2^{\text {nd }}$ and $3^{\text {rd }}$. It behaves better than the $4^{\text {th }}$ priority because there are no more priorities that could block the transmission in favor of a higher priorities. At the other hand, it behaves worst than the $2^{\text {nd }}$ and $3^{\text {rd }}$ priorities because it has 3 times their stand alone traffic profile: when $2^{\text {nd }}$ and $3^{\text {rd }}$ priority occupy $20 \%$ of the total load each, the "all" priority occupies $60 \%$. The delay is increased grammically with the total load until the $90 \%$ point, from where the systems starts to saturate. The "all" line seems to increase asymptotically to the $100 \%$ limit. 
However, the sources used do not model higher layers where the typical congestion avoidance of TCP would exercise flow control in response to losses and reduce the rate, while re-transmitting lost packets. So the system thanks to prioritization can guarantee the performance enjoyed by sensitive traffic while exploiting any unreserved bandwidth for the support of besteffort traffic.

\section{CONCLUSION}

Tree-shaped shared medium access networks such as PONs and HFC effect a distributed multiplexing function which concentrates traffic from many users and many services with diverse requirements. To be able to guarantee that the QoS of sensitive traffic will not be disturbed by best-effort data traffic requires embedding differentiated support for flow aggregates with common requirements. The architecture considered in this paper is quite advantageous in this context and a MAC protocol taking advantage of this approach was implemented and evaluated with satisfactory results.

\section{ACKNOWLEDGEMENT:}

The work presented in this paper was partially funded by the EU ACTS project AC327 "AROMA". The opinions appearing here are those of the authors and not necessarily of the other members of the AROMA consortium.

\section{REFERENCES}

[1] IETF, Differentiated Services Working Group, RFC 2475 “Architecture for Differentiated Services", December 1998

[2] Van Jacobson, Kathleen Nichols, Kedarnath Poduri, Internet Draft, draft-ietf-diffserv-phbef-02.txt, “An Expedited Forwarding PHB”, February, 1999

[3] Juha Heinanen, Fred Baker, John Wroclawski, Internet Draft, draft-ietf-diffserv-af-06.txt "Assured Forwarding PHB Group", February, 1999

[4] IEEE 802.14 MAC Draft(R3) Specifications, May 1997 (not publicly available).

[5] J.D.Angelopoulos, G.C. Boukis, I.S.Venieris, "Delay priorities enhance utilization of ATM PON Access Systems", Computer Communications Journal, Elsevier, Vol. 20, No. , December 1997, pp. 937-949.

[6] J. D. Angelopoulos, Th. Orphanoudakis, "An ATM-friendly MAC for traffic concentration in HFC systems", Computer Communications Journal, Elsevier, Vol. 21, No. 6, 25 May 1998, pp. 516-529. 
[7] J. D. Angelopoulos, N. I. Lepidas, E. K. Fragoulopoulos, I.S. Venieris, “TDMA multiplexing of ATM cells in a residential access SuperPON”, IEEE Journal on Selected Areas in Comm., Special issue on high capacity optical transport networks, Vol. 16, No. 7, September, 1998.

[8] ATM Forum Technical Committe, Traffic Management Group, "ATM Forum Traffic Management Specification Version 4.0" Doc. 95-013R11, March 1996.

[9] ITU-TS Rec. G.983, "High speed optical access systems based on PON techniques" SG15, Feb. 1998. 